\title{
Adult-onset Mitochondrial Myopathy, Encephalopathy, Lactic Acidosis, and Stroke (MELAS)-like Encephalopathy Diagnosed Based on the Complete Sequencing of Mitochondrial DNA Extracted from Biopsied Muscle without any Myopathic Changes
}

\author{
Masako Mukai ${ }^{1}$, Eiichiro Nagata ${ }^{1}$, Atsushi Mizuma ${ }^{1}$, Mitsuhiko Yamano $^{1}$, Keizo Sugaya ${ }^{2}$, \\ Ichizo Nishino $^{3}$, Yu-ichi Goto ${ }^{4}$ and Shunya Takizawa ${ }^{1}$
}

\begin{abstract}
The clinical features of mitochondrial myopathy, encephalopathy, lactic acidosis, and stroke-like episodes (MELAS) are not uniform. We herein report a male patient with unusual MELAS-like encephalopathy who had been experiencing isolated recurrent stroke-like episodes since he was 33 years old without any particular family history. Despite an extensive investigation, he had no other signs suggestive of MELAS. Although the muscle pathology showed a normal appearance, a mitochondrial genome sequence analysis of the biopsied muscle revealed a heteroplasmic m.10158T $>\mathrm{C}$ mutation in the mitochondrial complex I subunit gene, $M T$ ND3. To prevented further deterioration of the higher brain function, the early diagnosis and treatment of mitochondrial stroke-like episodes is important.
\end{abstract}

Key words: MELAS, stroke-like episodes, MT-ND3, adult onset, m.10158T>C mutation

(Intern Med 56: 95-99, 2017)

(DOI: 10.2169/internalmedicine.56.7301)

\section{Introduction}

Mitochondrial myopathy, encephalopathy, lactic acidosis, and stroke-like episodes (MELAS) syndrome is a multisystem mitochondrial disorder characterized by stroke-like episodes with various combinations of additional symptoms. The diagnosis of MELAS is usually based on characteristic symptoms such as lactic acidosis, deafness, diabetes mellitus, short stature, myopathy, and cognitive decline, often with a family history showing maternal inheritance. We herein report an unusual case of MELAS-like encephalopathy with isolated recurrent stroke-like episodes in adulthood. The diagnosis in this case was supported by the detection of a mitochondrial MT-ND3 gene mutation.

\section{Case Report}

The patient was a 41-year-old man who had experienced generalized convulsions, insomnia, and depression since 20 years of age. He had been diagnosed with ischemic stroke at 33 years of age and epileptic seizure with epileptic encephalopathy at 37 years of age. He had no other systemic signs of mitochondrial disorder, such as short stature, diabetes mellitus, deafness or heart failure. His family history was not remarkable. He developed myoclonus in the distal extremities, gait disturbance, and dysarthria at 41 years of age. Three months later, he was admitted to our hospital due to impaired consciousness and vomiting.

A neurological examination showed gaze nystagmus, cerebellar ataxia, and myoclonic movement in his distal extremi-

\footnotetext{
${ }^{1}$ Department of Neurology, Tokai University School of Medicine, Japan, ${ }^{2}$ Department of Neurology, Tokyo Metropolitan Neurological Hospital, Japan, ${ }^{3}$ Department of Neuromuscular Research, National Institute of Neuroscience, National Center of Neurology and Psychiatry, Japan and ${ }^{4}$ Medical Genome Center, National Center of Neurology and Psychiatry, Japan Received for publication February 24, 2016; Accepted for publication May 16, 2016 Correspondence to Dr. Masako Mukai, mmukai@tokai.ac.jp
} 
ties. However, no ophthalmoplegia, hearing loss, or muscle weakness were detected. His mini-mental status examination score was 15/30, and his frontal assessment battery score was 5/18. The findings from routine blood tests including blood sugar and autoimmune antibodies were normal. A laboratory examination showed slightly increased serum pyruvic acid levels $(1.2 \mathrm{mg} / \mathrm{dL})$, but his serum lactate levels were normal $(14.2 \mathrm{mg} / \mathrm{dL})$. The cerebrospinal fluid lactate $(34.8 \mathrm{mg} / \mathrm{dL})$ and pyruvic acid $(1.6 \mathrm{mg} / \mathrm{dL})$ levels were elevated. Electrocardiogram and echocardiogram were normal. Brain magnetic resonance imaging (MRI) showed bilateral cortical and subcortical high-intensity lesions on T2weighted imaging (T2WI) and fluid attenuated inversion recovery (FLAIR), distributed bilaterally and almost symmetrically. Bilateral red nuclei, mesencephalic tectum, vermis and cerebellar flocculus were also involved (Fig. 1A and C). Some of the cortical and subcortical lesions gave a high signal on diffusion-weighted imaging (DWI) (Fig. 1B). These lesions appeared as high or iso signal intensity on the apparent diffusion coefficient (ADC) map.

Two weeks later, the high signal on DWI had diminished, and four weeks later, the high signal on T2WI and FLAIR had diminished (Fig. 2). ${ }^{1} \mathrm{H}-\mathrm{MRS}$ revealed elevated lactate concentrations in the lesions (Fig. 1D). ${ }^{99 \mathrm{~m}} \mathrm{Tc}-$ Ethylcysteinate dimer single photon emission computed tomography (SPECT) imaging revealed bilateral multifocal increase of perfusion in the MRI lesions (Fig. 1E). We suspected mitochondrial encephalopathy, such as MELAS. A histopathological study of the biopsied right biceps branchii muscle revealed mild variation in the fiber size, measuring from 30 to 90 microns in diameter, which was thought to be a nonspecific change. No necrotic or regenerating fibers were seen. On modified Gomori-trichrome stain, ragged red fibers (RRFs) were not observed. Succinate dehydrogenase (SDH) stain revealed no strongly SDH-reactive blood vessels. Cytochrome $\mathrm{c}$ oxidase staining revealed no abnormalities (Fig. 1F). However, the complete sequencing of mitochondrial DNA samples extracted from the biopsied muscle revealed a heteroplasmic m.10158T $>$ C mutation, with a level of mutant heteroplasmy of $69.5 \%$, in the mitochondrial complex I subunit gene, MT-ND3 (Fig. 1G).

Because his family had no clinical symptoms suggestive of mitochondrial diseases, we could not conduct a complete mtDNA sequence analysis of his family. Over a two-month period, he experienced two stroke-like episodes with simple partial seizure and vomiting. Each time the stroke-like episodes ended within four days with drip infusion of edaravone and supportive care. To control epileptic seizure, we added levetiracetam (1,000 mg/day) to zonisamide $(400 \mathrm{mg} /$ day) and carbamazepine (400 mg/day). Because he had not yet been diagnosed with MELAS-like encephalopathy at that time, we did not administer L-arginine. His higher brain dysfunction and myoclonus worsened with each stroke-like episode.

\section{Discussion}

We herein report an unusual adult case of MELAS-like encephalopathy where the diagnosis was delayed because the adult-onset isolated stroke-like episodes were not accompanied with a family history showing either maternal inheritance or symptoms such as deafness, diabetes mellitus, heart failure, short stature, myopathy, or mental retardation. In addition, a histopathological study of the biopsied muscle revealed no myopathic features or RRFs. However, a sequence analysis of the mitochondrial genome extracted from the biopsied muscle revealed a heteroplasmic m.10158T $>\mathrm{C}$ mutation in the mitochondrial complex I subunit gene, MT-ND3.

Stroke-like episodes are recurrent neurological deficits resembling vaso-occlusive strokes. Stroke-like lesions may evolve subacutely over hours to days but have greater potential for reversibility than actual strokes. Recent studies have demonstrated a beneficial effect of L-arginine in MELAS for the acute treatment and prevention of stroke-like episodes (1). Indeed, we previously reported a woman harboring a m.10158T $>C$ mutation who was successfully treated with L-arginine and anti-epileptic agents (2) (Table). She developed right-side hemianopia and motor aphasia at 63 years of age, which was her first stroke-like episode. She experienced four additional stroke-like episodes in the year after the onset. Her aphasia worsened with each episode but completely recovered to baseline with radiological improvement following treatment with high-dose L-arginine in each acute phase. At the three-year follow-up, she had no neurological deficit except for minimal word-finding difficulties and appeared otherwise healthy. These features are clearly different from the progressive nature of MELAS and may be due to the lack of multi-systemic features in MELAS.

This lack of multi-systemic features in MELAS and the atypical brain MRI findings in the present case resulted in a delayed diagnosis. He was not developmentally disabled and had been healthy until he developed insomnia and depression at 20 years of age. When he was admitted to our hospital at 41 years of age, he was suffering from higher brain dysfunction. His higher brain functions and neurological manifestations worsened with each stroke-like episode, resulting in serious physical and cognitive sequelae. On comparing these two adult-onset cases harboring m.10158T $>C$, the early diagnosis and treatment for 'mitochondrial' strokelike episodes, especially L-arginine therapy, may help prevent further deterioration, particularly of the higher brain function.

The m.10158T $>\mathrm{C}$ mutation, which is known to cause lethal infant-onset Leigh syndrome, is located within the coding region of the loop domain of the ND3 subunit protein. The m.10158T $>\mathrm{C}$ mutation has been reported to decrease complex I activity to $8-23 \%$ of that of controls $(3,4)$. The common clinical features related to the m.10158T $>C$ mutation in a total of 10 patients, including our 2 adult-onset cases as well as previously described infant and pediatric 

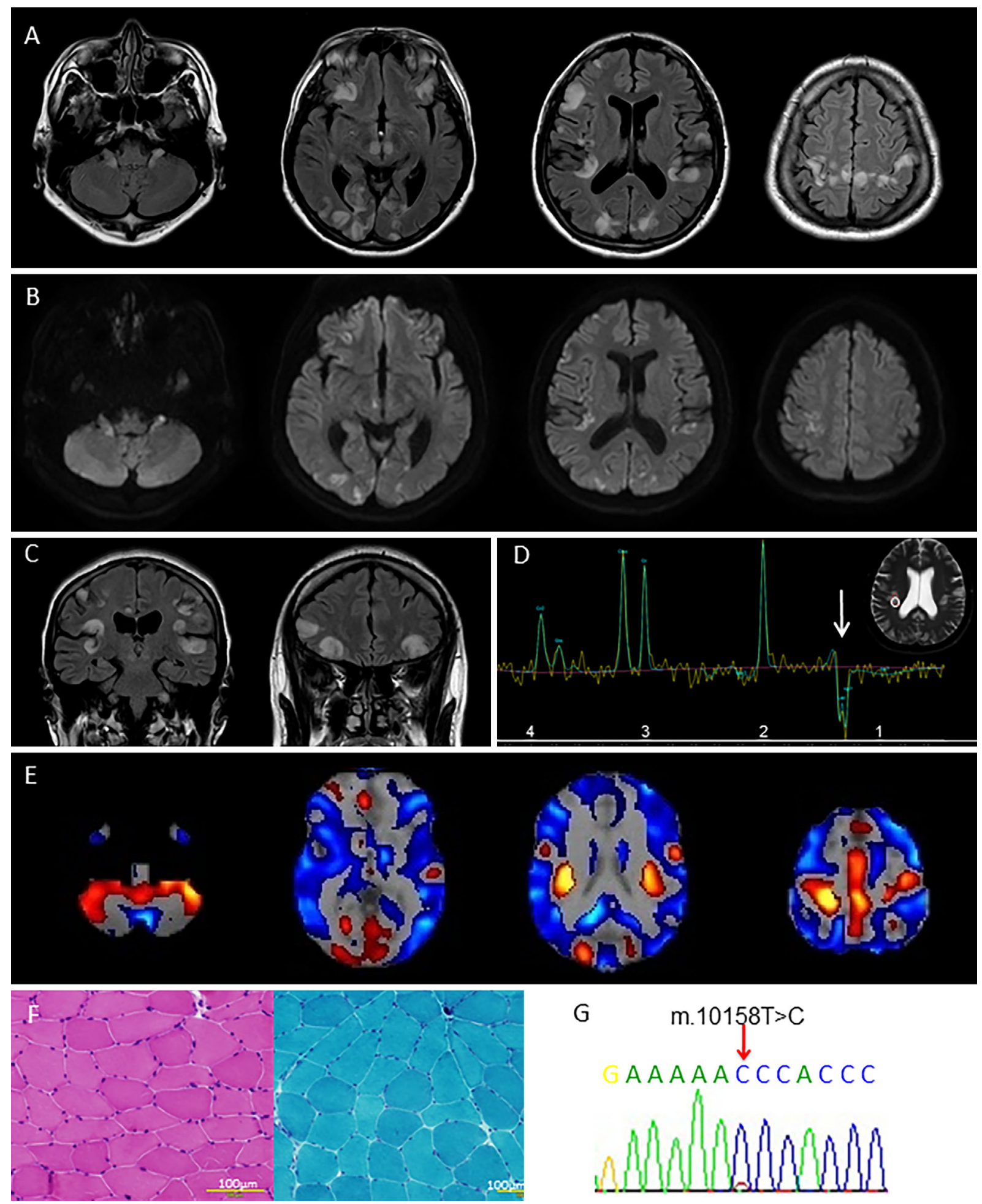

G

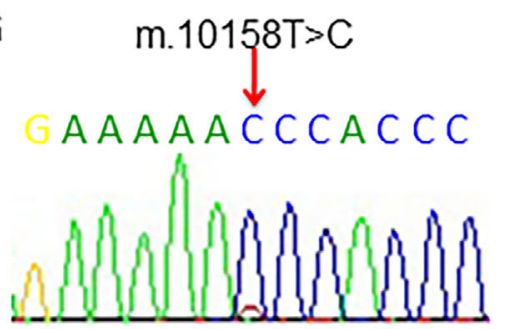

Figure 1. (A, C) Axial and coronal FLAIR brain MRI images showing cortical and subcortical high-intensity lesions distributed bilaterally and almost symmetrically. (B) Some of the cortical and subcortical lesions gave a high signal on diffusion-weighted imaging. (D) A large lactate doublet (arrow) was identified on MRS centered in the lesion. (E) ${ }^{99 \mathrm{~m}}$ Tc-Ethylcysteinate dimer SPECT imaging revealed a bilateral multifocal increase in the perfusion of the cortical lesions. (F) A histopathological study of the biopsied muscle showed no myopathic changes or ragged red fibers on Hematoxylin and Eosin staining and Gomori trichrome staining. (G) Sequence chromatogram of mitochondrial DNA extracted from the biopsied muscle. A heteroplasmic substitution $(\mathrm{m.10158T}>\mathrm{C})$ was found in the MT-ND3 gene (arrow). 


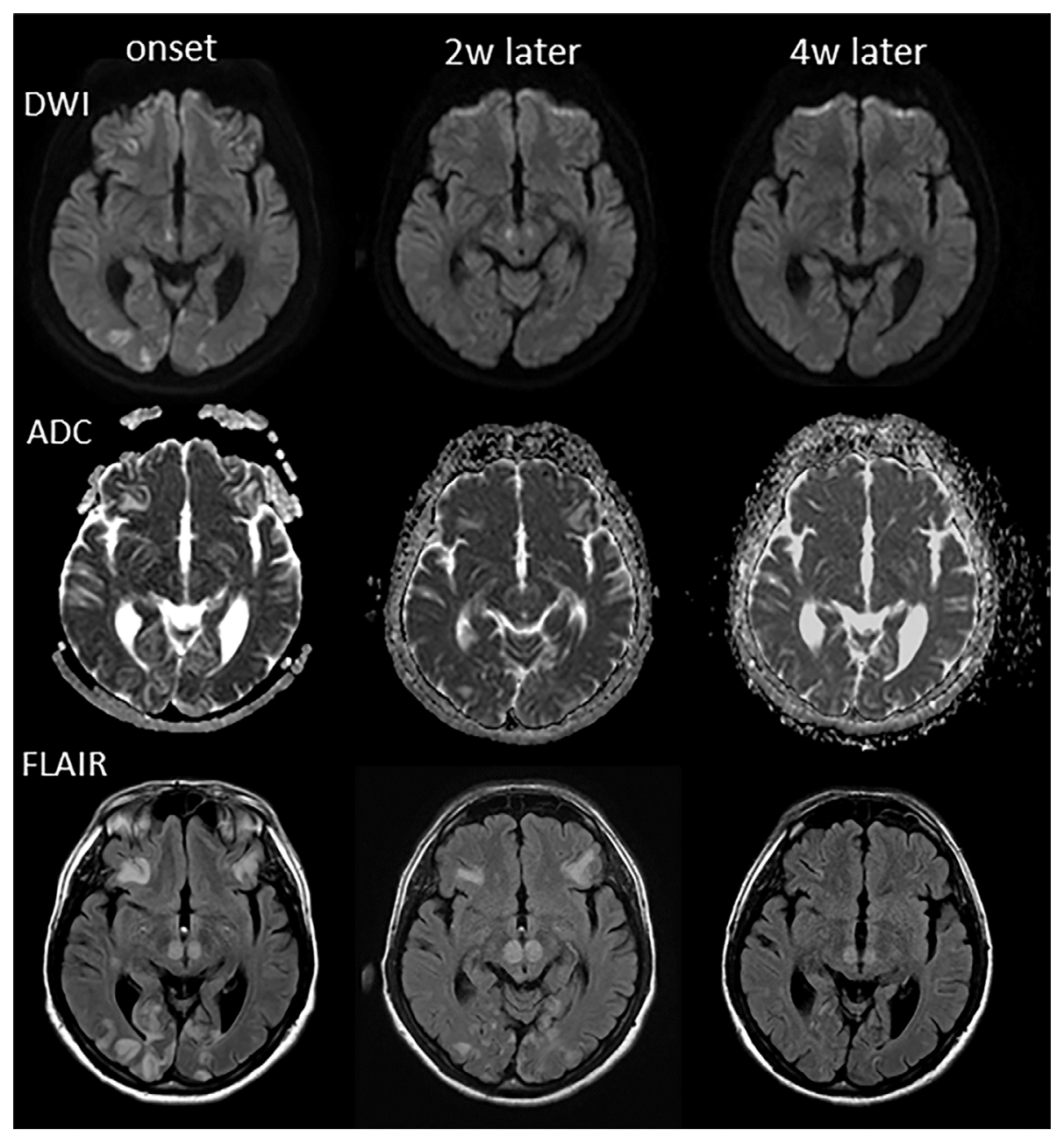

Figure 2. Brain MRI of DWI (upper), ADC map (middle), and FLAIR (bottom) images are shown. The high-intensity lesions diminished after 2 weeks on DWI. Four weeks later, some lesions still remained on FLAIR images.

patients, are a relatively high rate of sporadic occurrence, selective vulnerability of the central nervous system (CNS), no myopathic findings, and no RRFs or cytochrome c oxidase (COX)-defective fibers, even in patients with relatively high mutation loads (2-7). The mutation load has been reported to vary among patients and specimens: muscle, blood, hair and fibroblasts. All of the patients showed a higher mutation load in the muscles (73-100\%) and a lower load in the blood (0-48\%) (3-7). Therefore, to detect diagnostic pathogenic mutations, not only blood but also biopsied muscle should be analyzed. Such heteroplasmy may account for the differences in the phenotypical expression, as patients harboring $\mathrm{m} .10158 \mathrm{~T}>\mathrm{C}$ mutation appear not to have non-neurological symptoms or relevant family history.

Despite the high mutation loads (from $70 \%$ to more than $98 \%$ ), a histological analysis of the biopsied muscle revealed no specific findings in the present or previous adult cases or in the previously reported infant harboring the m.10158T $>\mathrm{C}$ mutation (3-7). RRFs have been found in up to $90 \%$ of MELAS patients, which is thought to be the histological hallmark of MELAS (8). A higher proportion of heteroplasmic mitochondrial DNA mutation was reported to be associ- ated with RRFs, based on an analysis of patients with different large mitochondrial DNA deletions and MELAS patients with the m.3243A $>\mathrm{G}$ point mutation (9). In addition to the m.10158T $>C$ mutation, the m.10191T $>C$ as well as m.10197G $>$ A mutations are located in the matrix-loop domain of the ND3 subunit protein and are known to cause Leigh syndrome or Leigh-like syndrome with dystonia, respectively $(6,10-12)$. Patients aged 16, 25, and 52 years harboring $73 \%, 70 \%$, and $77 \%$ m.10191T $>C$ mutation in muscle $(6,10,11)$ and 9-month-old and 37-year-old patients harboring $100 \%$ and $97 \% \mathrm{~m} .10197 \mathrm{G}>\mathrm{A}$ mutation (12) were found to have no RRFs, although all of these patients who underwent an analysis of mitochondrial respiratory chain activities in muscle showed a specific defect in the activity of complex I. It seems to be unlikely that the pathological analysis of the muscle was conducted too early to show any pathological changes. Thus, the absence of any pathological changes in the biopsied muscles despite a high mutation load and the predominance of CNS manifestations might be common among patients harboring pathogenic mutations in the ND3 gene.

Although L-arginine has emerged as an effective treat- 


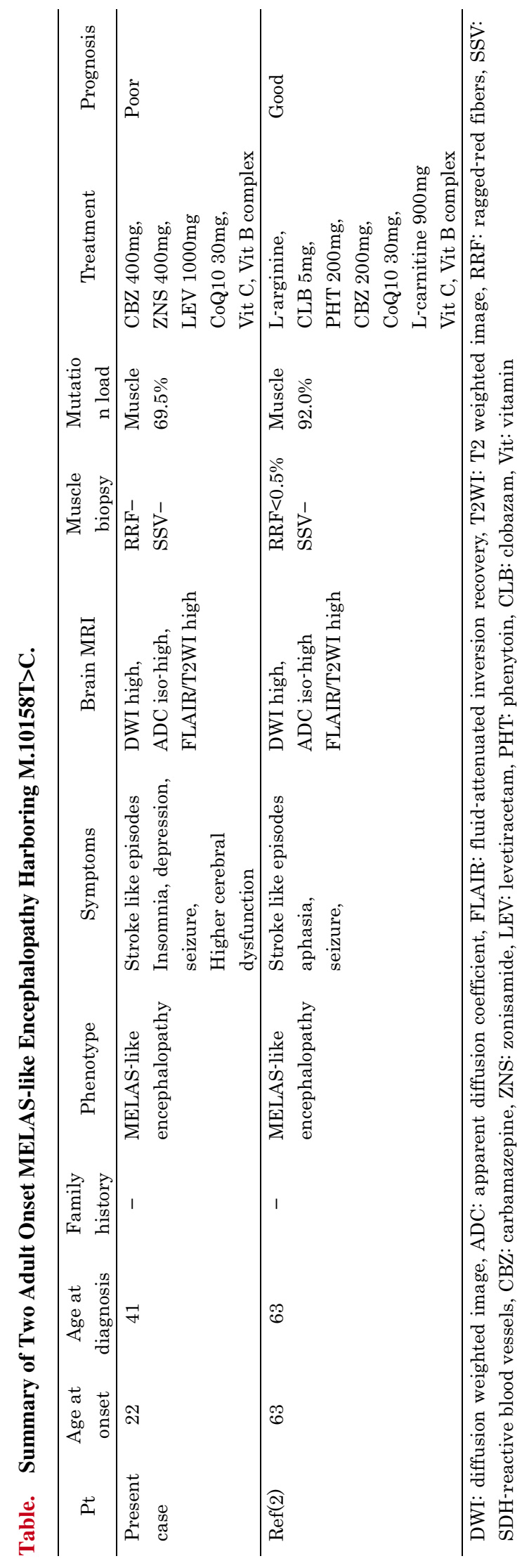

ment of stroke-like episodes in MELAS, the unique manifestation of adult-onset, isolated 'mitochondrial' stroke-like episodes in our patients could be easily misdiagnosed, resulting in serious sequelae. Mitochondrial DNA analyses using biopsied muscle should be considered in patients with suspected stroke-like episodes, even if the muscle pathology shows a normal appearance.

The authors state that they have no Conflict of Interest (COI).

\section{References}

1. Mukai M, Sugaya K, Ozawa $T$, et al. Isolated mitochondrial stroke-like episodes in an elderly patient with the MT-ND3 gene mutation. Neurol Clin Neuroscience 3: 153-156, 2015.

2. Koga Y, Akita Y, Nishioka J, et al. L-arginine improves the symptoms of strokelike episodes in MELAS. Neurology 64: 710-712, 2005.

3. McFarland R, Kirby DM, Fowler KJ, et al. De novo mutations in the mitochondrial ND3 gene as a cause of infantile mitochondrial encephalopathy and complex I deficiency. Ann Neurol 55: 58-64, 2004.

4. Crimi M, Papadimitriou A, Galbiati S, et al. A new mitochondrial DNA mutation in ND3 gene causing severe Leigh syndrome with early lethality. Pediatr Res 55: 842-846, 2004.

5. Lebon S, Chol M, Benit P, et al. Recurrent de novo mitochondrial DNA mutations in respiratory chain deficiency. J Med Genet 40: 896-899, 2003.

6. Bugiani M, Invernizzi F, Alberio S, et al. Clinical and molecular findings in children with complex I deficiency. Biochim Biophys Acta 1659: 136-147, 2004.

7. Werner KG, Morel CF, Kirton A, et al. Rolandic mitochondrial encephalomyelopathy and MT-ND3 mutations. Pediatr Neurol 41: 27-33, 2009.

8. Goto Y, Horai S, Matsuoka T, et al. Mitochondrial myopathy, encephalopathy, lactic acidosis, and stroke-like episodes (MELAS): a correlative study of the clinical features and mitochondrial DNA mutation. Neurology 42: 545-550, 1992.

9. Auré K, Fayet G, Leroy JP, et al. Apoptosis in mitochondrial myopathies is linked to mitochondrial proliferation. Brain 129: 1249-1259, 2006.

10. Levy RJ, Ríos PG, Akman HO, et al. Long survival in patients with Leigh syndrome and the m.10191T>C mutation in MT-ND3 : a case report and review of the literature. J Child Neurol 29: NP 105-NP110, 2014.

11. Taylor RW, Singh-Kler R, Hayes CM, et al. Progressive mitochondrial disease resulting from a novel missense mutation in the mitochondrial DNA ND3 gene. Ann Neurol 50: 104-107, 2001.

12. Sarzi E, Brown MD, Lebon S, et al. A novel recurrent mitochondrial DNA mutation in ND3 gene is associated with isolated complex I deficiency causing Leigh syndrome and dystonia. Am J Med Genet A 143: 33-41, 2007.

The Internal Medicine is an Open Access article distributed under the Creative Commons Attribution-NonCommercial-NoDerivatives 4.0 International License. To view the details of this license, please visit (https://creativecommons.org/licenses/ by-nc-nd/4.0/).

(C) 2017 The Japanese Society of Internal Medicine http://www.naika.or.jp/imonline/index.html 